Pacific Journal of Mathematic

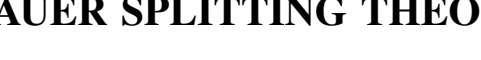




\section{ON THE BRAUER SPLITTING THEOREM}

\section{George Szeto}

This paper presents a proof for the Brauer splitting theorem in the context of a commutative ring with no idempotents except 0 and 1 and continues this investigation. The main results in this paper are the Brauer splitting theorem and the classification of all finitely generated projective indecomposable modules over a separable group algebra.

Throughout this paper we assume that the ring $R$ is a commutative ring with no idempotents except 0 and 1 , that the group $G$ has order $n$ invertible in $R$, and that all $R G$-modules are unitary left $R G$ modules. We know that the order of $G, n$, is invertible in $R$ if and only if $R G$ is separable.

1. First, let us recall the following Brauer splitting theorem: Let $K$ be a field and $G$ be a group of order $n$ invertible in $K$, then $K(\sqrt[m]{1})$ is a splitting field for $G$, where $m$ is the exponent of $G$ and $\sqrt[m]{1}$ is a primitive $m^{\text {th }}$-root of 1 ([6], Th. 41-1, p. 292 and Corollary $70-24$, p. 475). In [8], G. J. Janusz defined a ring $R$ to be a splitting ring for $G$ if the group algebra $R G$ is the direct sum of central separable $R$-algebras each equivalent to $R$ in the Brauer group of $R$; that is, $R G \cong \bigoplus \sum_{i=1}^{s} \operatorname{Hom}_{R}\left(P_{i}, P_{i}\right)$, where $\left\{P_{i}\right\}$ are finitely generated projective faithful $R$-modules, the number of different conjugate classes in $G$ is equal to $s$. He then proved the Brauer splitting theorem for a Noetherian regular domain, $R$. This section gives a proof for the above theorem when $R$ is any commutative ring with no idempotents except 0 and 1.

LEMma 1. Let $R_{0}$ be a subring of $R$. If $R_{0}$ is a splitting ring for $G$, then $R$ is a splitting ring for $G$.

Proof. Because $R_{0}$ is a splitting ring for $G, R_{0} G \cong \bigoplus \sum_{i=1}^{s} \operatorname{Hom}_{R_{0}}$ $\left(P_{i}, P_{i}\right)$ where $\left\{P_{i}\right\}$ are finitely generated projective faithful $R_{0}$-modules. Then we have

$$
\begin{aligned}
R G & \cong R \bigotimes_{R_{0}} R_{0} G \cong R \bigotimes_{R_{0}}\left(\oplus \sum_{i=1}^{s} \operatorname{Hom}_{R_{0}}\left(P_{i}, P_{i}\right)\right) \\
& \cong \oplus \sum_{i=1}^{s} R \bigotimes_{R_{0}} \operatorname{Hom}_{R_{0}}\left(P_{i}, P_{i}\right) \cong \bigoplus \sum_{i=1}^{s} \operatorname{Hom}_{R}\left(R \bigotimes_{R_{0}} P_{i}, R \bigotimes_{R_{0}} P_{i}\right),
\end{aligned}
$$

where $\left\{R \otimes_{R_{0}} P_{i}\right\}$ are finitely generated projective faithful $R$-modules. This follows since $\left\{P_{i}\right\}$ are finitely generated projective faithful $R_{0^{-}}$ 
modules ([1], Proposition 5-5). Thus $R$ is a splitting ring for $G$.

THEOREM 2. If $R$ is a commutative ring with no idempotents except 0 and 1 and $R G$ is a separable group algebra, then $R[\sqrt[m]{1}]$ is a splitting ring for $G$ where $\sqrt[m]{1}$ is a primitive $m^{\text {th }}$-root of 1 .

Proof. Let $Z$ be the set of integers, $Q$ be the set of rationals. The proof divides into two cases.

Case 1. The prime ring of $R$ is finite. Let Char $(R)=p^{e}$, where $p$ is a prime integer and $e$ is in $Z$.

$Z /\left(p^{e}\right)$ is a local ring with the maximal ideal $(p) /\left(p^{e}\right)$ which is also nilpotent. For $\left(Z /\left(p^{e}\right)\right)[\theta]$ where $\theta=\sqrt[m]{1}$, we have

$$
\frac{\left(Z /\left(p^{e}\right)\right)[\theta]}{\left((p) /\left(p^{e}\right)\right)[\theta]} \cong(Z /(p))(\bar{\theta})
$$

where $\bar{\theta}$ is a primitive $m^{\text {th }}$-root of 1 over $Z /(p)$. Now $(Z /(p))(\bar{\theta})$ is a field; so $\left((p) /\left(p^{e}\right)\right)[\theta]$ is a maximal ideal. On the other hand, since $(p) /\left(p^{e}\right)$ is nilpotent, $\left((p) /\left(p^{e}\right)\right)[\theta]$ is also nilpotent. But then $\left((p) /\left(p^{e}\right)\right)[\theta]$ is an unique maximal ideal and a nilpotent ideal of $\left(Z /\left(p^{e}\right)\right)[\theta]$. Therefore, $\left(Z /\left(p^{e}\right)\right)[\theta]$ is a complete local ring where the completion is in the sense of $m$-topology (see [9], p. 254). Then the Brauer group natural map

$$
B\left(\left(Z /\left(p^{e}\right)\right)[\theta]\right) \longrightarrow B\left(\frac{\left(Z /\left(p^{e}\right)\right)[\theta]}{\left((p) /\left(p^{e}\right)\right)[\theta]}\right) \cong B((Z /(p))(\bar{\theta}))
$$

is monomorphic ([1], Corollary 6-2). But $(Z /(p))(\bar{\theta})$ is a splitting field for $G$; so $\left(Z /\left(p^{e}\right)\right)[\theta]$ is a splitting ring for $G$. Thus $R[\theta]$ is a splitting ring for $G$ by the lemma.

Case 2. The prime ring of $R$ is $Z(n)$ which is the quotient ring of $Z$ with respect to the multiplicative closed set $\left\{n, n^{2}, \cdots\right\}$. Since $Z(n)[\theta]$ is a Dedekind domain, it is Noetherian and regular. Then the Brauer group natural map $B(Z(n)[\theta]) \rightarrow B(Q(\theta))$ is monomorphic ([1], Th. 7-2). But $Q(\theta)$ is the quotient field of $Z(n)[\theta]$ and a splitting field for $G$ by the Brauer splitting theorem. Therefore, $Z(n)[\theta]$ is a splitting ring for $G$ and so $R[\sqrt[m]{1}]$ is a splitting ring for $G$ by the lemma. By combining Cases 1 and 2, the theorem is proved.

REMARK. The above theorem tells us the existence of a splitting ring, $R[\sqrt[m]{1}]$, for $G$, if $R G$ is a separable group algebra. We also know that $R[\sqrt[m]{1}]$ is a finitely generated projective and separable $R$ algebra ([8], Corollary 2-4). But there exists a central separable $R$ - 
algebra without a finitely generated projective and separable splitting ring. The following example is due to 0 . Goldman: Let $R$ be $Z[\sqrt{2}], i, j, k$ be the usual quaternion basis. If $\alpha=(1+i) / \sqrt{2}$ and $\beta=(1+j) / \sqrt{2}$, then $R 1 \oplus R \alpha \oplus R \beta \oplus R \alpha \beta$ is central separable over $R$. But $R$ has no finitely generated projective and separable extension except direct sums of copies of $R$, and $R 1 \oplus R \alpha \oplus R \beta \oplus R \alpha \beta$ cannot be split.

2. In this section, assume $R G$ is a split group algebra,

$$
R G \cong \oplus \sum_{i} \operatorname{Hom}_{R}\left(P_{i}, P_{i}\right), \quad i=1,2, \cdots, s .
$$

When $\left\{P_{i}\right\}$ are considered as $R G$-modules ([3], p. 5), the classification of all finitely generated projective indecomposable $R G$-modules can be obtained. Observe that the order of the group $G, n$, is invertible in $R$ if and only if $R G$ is separable. Therefore, any $R G$-module $M$ is finitely generated and projective over $R G$ if and only if $M$ is finitely generated and projective over $R$ (see the proof of Proposition 1-5 in [8]).

Let $R G$ be a separable $R$-algebra and $M$ be a finitely generated projective $R G$-module; for any $x$ in $M$ there exist $X_{1}, X_{2}, \cdots X_{q}$ in $M$ and $F_{1}, F_{2}, \cdots, F_{q}$ in $\operatorname{Hom}_{R}(M, R)$ so that $x=\sum_{i=1}^{q} F_{i}(x) X_{i}$. We call $\left\{F_{i}, X_{i}, i=1,2, \cdots, q\right\}$ a $R$-dual basis of $M$, and $T_{M}(x)=\sum_{i=1}^{q} F_{i}\left(x X_{i}\right)$ the character of $M$ at $x$ in $R G$ ([4], Proposition 3-1). By a group character we mean the restriction of $T_{M}$ to $G$. Obviously, a character $T_{M}$ is completely determined by its restriction to $G$. In particular, let $R$ be a splitting ring for $G$; then

$$
R G \cong \bigoplus \sum_{i=1}^{s} \operatorname{Hom}_{R}\left(P_{i}, P_{i}\right) \cong \bigoplus \sum_{i=1}^{s}(R G) E_{i},
$$

where $E_{i}$ is the $i^{\text {th }}$-central primitive idempotent of $R G$. We let

$$
T^{i}=T_{P_{i}} .
$$

Proposition 3. If $M$ and $N$ are two isomorphic finitely generated projective RG-modules, then they have the same characters.

Proof. Let $M$ and $N$ be two isomorphic finitely generated projective $R G$-modules and let $\alpha$ be the isomorphism. If $\left\{F_{i}, X_{i}, i=1,2\right.$, $\cdots, q\}$ is a dual basis of $M$, then we claim that $\left\{F_{i} \alpha^{-1}, \alpha X_{i}, i=1,2\right.$, $\cdots, q\}$ is a dual basis of $N$. In fact, for any $a$ in $N$, there exists $b$ in $M$ such that $\alpha(b)=\alpha$; so

$$
\begin{aligned}
a & \left.=\alpha\left(\sum_{i=1}^{a} F_{i}(b) X_{i}\right)\right)=\sum_{i} F_{i}(b)\left(\alpha X_{i}\right) \\
& =\sum_{i} F_{i} \alpha^{-1} \alpha(b)\left(\alpha X_{i}\right)=\sum_{i}\left(\left(F_{i} \alpha^{-1}\right) \alpha(b)\right)\left(\alpha X_{i}\right) \\
& =\sum_{i}\left(F_{i} \alpha^{-1}(a)\right)\left(\alpha X_{i}\right) .
\end{aligned}
$$


This means that $\left\{F_{i} \alpha^{-1}, \alpha X_{i}, i=1,2, \cdots, q\right\}$ is a dual basis of $N$. But the character of any finitely generated projective $R G$-module is independent of the dual basis chosen; so $T_{N}(g)=\sum_{i} F_{i} \alpha^{-1}\left(g \alpha X_{i}\right)=$ $\sum_{i} F_{i} \alpha^{-1}\left(\alpha g X_{i}\right)$, (for $\alpha$ is a $R G$-isomorphism), and so $=\sum_{i} F_{i}\left(g X_{i}\right)=$ $T_{M}(g)$.

The following proposition will play an important role in our discussion.

Proposition 4. If $N$ is a finitely generated projective faithful $R$-module and $M$ a finitely generated projective left $\operatorname{Hom}_{R}(N, N)$ module, then $M \cong N \bigotimes_{R} N^{\prime}$ with $N^{\prime}$ a finitely generated projective $R$-module.

Proof. By the Morita Theorem on p. 9 in [3].

REMark. Proposition 4 gives a counter-example to the converse statement of Proposition 3. Because of Proposition 4, let $M$ and $N$ be two finitely generated projective indecomposable $R G$-modules over the same central component of the split group algebra $R G$; that is, $\operatorname{Hom}_{R}\left(P_{i}, P_{i}\right)$, then $M \cong P_{i} \otimes_{R} N^{\prime}$ and $N \cong P_{i} \bigotimes_{R} N^{\prime \prime}$, where $N^{\prime}$ and $N^{\prime \prime}$ are finitely generated projective indecomposable $R$-modules. Suppose $N^{\prime}$ and $N^{\prime \prime}$ are in $P(R)$, the class group of $R$, then

$$
T_{y y}(g)=T_{P_{i}}(g) T_{N^{\prime}}(1)=T_{P_{i}}(g) \cdot 1=T_{N}(g) .
$$

But $P_{i} \otimes_{R} N^{\prime} \cong P_{i} \otimes_{R} N^{\prime \prime}$ only if $N^{\prime} \cong N^{\prime \prime}$.

Lemma 5. If RG is a split group algebra; that is,

$$
R G \cong \bigoplus \sum_{i=1}^{s} \operatorname{Hom}_{R}\left(P_{i}, P_{i}\right) \cong \bigoplus \sum_{i=1}^{s}(R G) E_{i},
$$

then

$$
E_{i}=\sum_{g} \frac{k_{i} T^{i}\left(g^{-1}\right)}{n} g
$$

where $g$ is in $G, k_{i}=\operatorname{rank}\left(P_{i}\right)$ and $T^{i}=T_{P_{i}}$.

Proof. Since

$$
R G \cong \bigoplus \sum_{i=1}^{s}(R G) E_{i} \cong \bigoplus \sum_{i=1}^{s} \operatorname{Hom}_{R}\left(P_{i}, P_{i}\right), E_{i}=\sum_{g} E_{i}(g) g,
$$

for all $g$ in $G, E_{i}(g)$ in $R$. We then have

$$
E_{i} h^{-1}=\sum_{g} E_{i}(g)\left(g h^{-1}\right)
$$


for some $h$ in $G$. Taking the character afforded by $R G$, we have

$$
T_{R G}\left(E_{i} h^{-1}\right)=\sum_{g} E_{i}(g) T_{R G}\left(g h^{-1}\right) .
$$

But $T_{R G}\left(g h^{-1}\right)=0$ in case $g h^{-1} \neq 1$, and $=n$ in case $g h^{-1}=1$ or $g=h$. Hence $T_{R G}\left(E_{i} h^{-1}\right)=E_{i}(h) n, E_{i}(h)=T_{R G}\left(E_{i} h^{-1}\right) / n$ (for $n$ is invertible in $R$ ).

Next, we find $T_{R G}\left(E_{i} h^{-1}\right)$. Because $P_{i}$ is a finitely generated projective $R$-module, $\operatorname{Hom}_{R}\left(P_{i}, P_{i}\right) \cong P_{i} \otimes_{R} \operatorname{Hom}_{R}\left(P_{i}, R\right)$ ([3], Morita Theorem I). Noting that $\operatorname{rank}\left(P_{i}\right)=\operatorname{rank}\left(\operatorname{Hom}_{R}\left(P_{i}, R\right)\right)$, we have

$$
T_{(R G) E_{i}}(g)=T^{i}(g) k_{i} \quad \text { for all } i=1,2, \cdots, s .
$$

Therefore,

$$
T_{R G}\left(E_{i} h^{-1}\right)=\sum_{j=1}^{s} T_{\left(R G^{\prime} E_{j}\right.}\left(E_{i} h^{-1}\right)=\sum_{j} k_{j} T^{j}\left(E_{i} h^{-1}\right) .
$$

But $T^{j}\left(E_{i} h^{-1}\right)=0$ in case $i \neq j$, so

$$
T_{R G}\left(E_{i} h^{-1}\right)=k_{i} T^{i}\left(E_{i} h^{-1}\right)=k_{i} T^{\imath}\left(h^{-1}\right) \text {. }
$$

Hence,

$$
E_{i}(h)=\frac{T_{R G}\left(E_{i} h^{-1}\right)}{n}=\frac{k_{i} T^{i}\left(h^{-1}\right)}{n} .
$$

By substituting $E_{i}(h)$ in $E_{i}$, we have

$$
E_{i}=\sum_{g} E_{i}(g) g=\sum_{g} \frac{k_{i} T^{i}\left(g^{-1}\right)}{n} g .
$$

This completes the proof.

Lemma 6. For $i=1,2, \cdots, s$, rank $\left(P_{i}\right)$ is neither 0 nor a zero divisor in $R$.

Proof. First, rank $\left(P_{i}\right)$ is not 0 , otherwise $E_{i}$ is 0 by Lemma 5. This is impossible.

Next, let rank $\left(P_{i}\right)$ be $k_{i}$, and suppose that $k_{i}$ is a zero divisor in $R$. We then have a nonzero element, $k^{\prime}$, in $R$ such that $k^{\prime} k=0$. But by Lemma 5 ,

$$
E_{i}=k_{i} \sum_{g} \frac{T^{i}\left(g^{-1}\right) g}{n}
$$

so,

$$
k^{\prime} E_{i}=k^{\prime} k_{i} \sum_{g} \frac{T^{i}\left(g^{-1}\right) g}{n}=\left(k^{\prime} k_{i}\right) \sum_{g} \frac{T^{i}\left(g^{-1}\right) g}{n}=0
$$


Noting that $(R G) E_{i} \cong \operatorname{Hom}_{R}\left(P_{i}, P_{i}\right)$, we have

$$
k^{\prime} \operatorname{Hom}_{R}\left(P_{i}, P_{i}\right) \cong k^{\prime}(R G) E_{i}=k^{\prime} E_{i}(R G)=0 .
$$

On the other hand, $P_{i}$ is a faithful $R$-module; so $\operatorname{Hom}_{R}\left(P_{i}, P_{i}\right)$ is a faithful $R$-module. Therefore, $k^{\prime} \operatorname{Hom}_{R}\left(P_{i}, P_{i}\right)=0$ implies $k^{\prime}=0$. This is a contradiction. Thus we have proved that $k_{i}$ is not a zero divisor in $R$.

Theorem 7, Suppose $R$ is a splitting ring for $G$ and all finitely generated projective indecomposable $R$-modules are of rank 1 . Then for any two finitely generated projective indecomposable $R G$-modules $M$ and $N$, we have $E_{i} M \neq 0$ and $E_{i} N \neq 0$ if and only if $T_{M}(g)=$ $T_{N}(g)$ for all $g$ in $G$, where $E_{i}$ is the $i^{\text {th }}$-central primitive idempotent of $R G$.

Proof. If $E_{i} M \neq 0$ and $E_{i} N \neq 0$, then $M \cong E_{i} M \oplus\left(1-E_{i}\right) M$ and $N \cong E_{i} N \oplus\left(1-E_{i}\right) N$. Since $M$ and $N$ are indecomposable, $\left(1-E_{i}\right) M=0$ and $\left(1-E_{i}\right) N=0$. We have $N=E_{i} N$ and $M=E_{i} M$ as left $\operatorname{Hom}_{R}\left(P_{i}, P_{i}\right)$-modules. Therefore, by Proposition $4, M \cong P_{i} \otimes_{I_{i}} N^{\prime}$ and $N \cong P_{i} \otimes_{R} N^{\prime \prime}$ where $N^{\prime}$ and $N^{\prime \prime}$ are finitely generated projective $R$-modules. Since $M$ and $N$ are indecomposable $R G$-modules, $N^{\prime}$ and $N^{\prime \prime}$ are in $P(R)$. Therefore,

$$
\begin{aligned}
T_{M I}(g) & =T_{P_{i} \otimes_{R^{N}}}(g)=T_{P_{i}}(g) \cdot 1 \\
& =T_{P_{i}}(g) T_{N^{\prime \prime}}(1)=T_{\Lambda^{\prime}}(g) .
\end{aligned}
$$

Conversely, if $T_{M I}(g)=T_{N}(g)$ for all $g$ in $G$, then $T_{M I}(a)=T_{N}(a)$ for all $a$ in $R G$. Suppose $E_{i} M \neq 0$ and $E_{i} N=0$ for some $i$; then there exists a $j \neq i$ such that $E_{j} N \neq 0$. Thus $M$ is a $(R G) E_{i}$-module and $N$ is a $(R G) E_{j}$-module, and so we have

$$
T_{M I}\left(E_{i}\right)=T_{P_{i}}\left(E_{i}\right)=T_{P_{i}}(1)=\operatorname{rank}\left(P_{i}\right) .
$$

By Lemma $6, \operatorname{rank}\left(P_{i}\right) \neq 0$ in $R$, so $T_{M}\left(E_{i}\right) \neq 0$. Obviously, $T_{\Lambda}\left(E_{i}\right)=0$. Thus $T_{M} \neq T_{N}$ on $R G$. Consequently, $T_{M}(g) \neq T_{\Lambda}(g)$ for some $g$ in $G$. This is a contradiction to $T_{M I}(g)=T_{N}(g)$ for all $g$ in $G$, and hence the proof is completed.

COROLLARY 8. If $R$ is a splitting ring for $G$, and all finitely generated projective indecomposable $R$-modules are of rank 1 ; then there are exactly s-classes of finitely generated projective indecomposable RG-modules over different central components each uniquel, determined up to an element in $P(R)$.

Proof. Let $M$ be a finitely generated projective indecomposable 
$R G$-module. From the theorem, we have $M=E_{i} M \cong P_{i} \bigotimes_{R} N^{\prime}$ where $N^{\prime}$ is in $P(R)$. On the other hand, $P_{i}, i=1,2, \cdots, s$, is a finitely generated projective indecomposable $R G$-module over the $i^{\text {th }}$-central component. Therefore, there are exactly s-classes of finitely generated projective indecomposable $R G$-modules each uniquely determined up to an element in $P(R)$.

From the above result, we have computed the first Grothendieck group of $R G, K^{0}(R G)$, in the sense of [2], p. 31 .

Corollary 9. If $R$ is a splitting ring for $G$, then

$$
K^{0}(R G)=(Z \oplus P(R)) \oplus(Z \oplus P(R)) \oplus \cdots \oplus(Z \oplus P(R)) .
$$

A natural question to ask is whether the classification of all finitely generated projective indecomposable $R G$-modules can be obtained for a nonsplit group algebra. The answer is not known. But for some special rings, we have a definite answer.

For a separable group algebra $R G$, we have the decomposition, $R G \cong \bigoplus \sum_{i=1}^{t} A_{i}$, where $A_{i}$ has no proper central idempotents and $t$ is an integer.

THEOREM 10. If $R$ is local or semi-local, then there are exactly $t$-isomorphic classes of finitely generated projective indecomposable $R G$-modules.

Proof. From the decomposition of $R G, A_{i}$ is a central separable $C_{i}$-algebra for each $A_{i}$, where $C_{i}$ is the center of $A_{i}$ ([1], Th. 2-3). Since $R$ is local or semi-local, $C_{i}$ is semi-local by the lemma on p. 25 in [5]. Therefore any two finitely generated projective indecomposable $R G$-modules over the $i^{t h}$-component $A_{i}$ are in an isomorphic class of finitely generated projective indecomposable $R G$-modules ([7], Th. 1).

COROLlary 11. If $R$ is local or semi-local, then

$$
K^{\circ}(R G)=Z \oplus Z \oplus \cdots \oplus Z,
$$

$t$-copies of $Z$.

This paper forms a part of the author's Doctoral Dissertation at Purdue University written under the guidance of Professor F. R. DeMeyer. The author wishes to thank Professor DeMeyer for his guidance, criticism, and encouragement throughout this study.

The author wishes to thank the referee for many helpful suggestions. 


\section{BIBLIOGRAPHY}

1. M. Auslander and O. Goldman, The Brauer group of a commutative ring, Trans. Amer. Math Soc. 97 (1960), 367-409.

2. H. Bass, K-theory and stable algebra, Publ. Math. I.H.E.S. Paris, 22 (1964), 5-60.

3. - The Morita theorems, Mimeographed Notes, University of Oregon, 1964.

4. H. Cartan and S. Eilenberg, Homological algebra, Princeton, 1956.

5. L. N. Childs and F. R. DeMeyer, On automorphisms of separable algebras, Pacific J. Math. 23 (1967), 25-34.

6. C. W. Curtis and I. Reiner, Representation theory of finite groups and associated Algebras, New York, Interscience, 1962.

7. F. R. DeMeyer, Projective modules over central separable algebras, Canad. J. Math. 21 (1969), 39-43.

8. G. J. Janusz, Separable algebra over commutative rings, Trans. Amer. Math. Soc. 122 (1966), 461-479.

9. O. Zariski and P. Samuel, Commutative algebra, Vol. 2, Princeton, D. Van Nostrand Co., 1960.

Received October 14, 1968.

BRADLEY UNIVERSITY

PEORIA, Illinois 


\section{PACIFIC JOURNAL OF MATHEMATICS}

\section{EDITORS}

\author{
H. ROYDEN \\ Stanford University \\ Stanford, California \\ Richard Pierce \\ University of Washington \\ Seattle, Washington 98105
}

\author{
J. DugundJI \\ Department of Mathematics \\ University of Southern California \\ Los Angeles, California 90007 \\ BASIL GoRDON \\ University of California \\ Los Angeles, California 90024
}

\section{ASSOCIATE EDITORS}
E. F. BECKENBACH
B. H. Neumann
F. WOLF
K. YoSHIDA

\section{SUPPORTING INSTITUTIONS}

\author{
UNIVERSITY OF BRITISH COLUMBIA \\ CALIFORNIA INSTITUTE OF TECHNOLOGY \\ UNIVERSITY OF CALIFORNIA \\ MONTANA STATE UNIVERSITY \\ UNIVERSITY OF NEVADA \\ NEW MEXICO STATE UNIVERSITY \\ OREGON STATE UNIVERSITY \\ UNIVERSITY OF OREGON \\ OSAKA UNIVERSITY \\ UNIVERSITY OF SOUTHERN CALIFORNIA
}

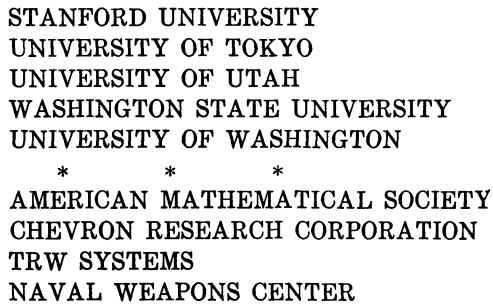

The Supporting Institutions listed above contribute to the cost of publication of this Journal, but they are not owners or publishers and have no responsibility for its content or policies.

Mathematical papers intended for publication in the Pacific Journal of Mathematics should be in typed form or offset-reproduced, double spaced with large margins. Underline Greek letters in red, German in green, and script in blue. The first paragraph or two must be capable of being used separately as a synopsis of the entire paper. It should not contain references to the bibliography. Manuscripts, in duplicate if possible, may be sent to any one of the four editors. Please classify according to the scheme of Math. Rev. 36, 1539-1546. All other communications to the editors should be addressed to the managing editor, Richard Arens, University of California, Los Angeles, California, 90024.

50 reprints are provided free for each article; additional copies may be obtained at cost in multiples of 50 .

The Pacific Journal of Mathematics is published monthly. Effective with Volume 16 the price per volume (3 numbers) is $\$ 8.00$; single issues, $\$ 3.00$. Special price for current issues to individual faculty members of supporting institutions and to individual members of the American Mathematical Society: $\$ 4.00$ per volume; single issues $\$ 1.50$. Back numbers are available.

Subscriptions, orders for back numbers, and changes of address should be sent to Pacific Journal of Mathematics, 103 Highland Boulevard, Berkeley, California, 94708.

PUBLISHED BY PACIFIC JOURNAL OF MATHEMATICS, A NON-PROFIT CORPORATION

Printed at Kokusai Bunken Insatsusha (International Academic Printing Co., Ltd.), 7-17, Fujimi 2-chome, Chiyoda-ku, Tokyo, Japan. 


\section{Pacific Journal of Mathematics}

\section{Vol. 31, No. $2 \quad$ December, 1969}

Efraim Pacillas Armendariz, Quasi-injective modules and stable torsion

classes..........................................

J. Adrian (John) Bondy, On Ulam's conjecture for separable graphs...

Vasily Cateforis and Francis Louis Sandomierski, On commutative rings over which the singular submodule is a direct summand for every module .....

Rafael Van Severen Chacon, Approximation of transformations with continuous

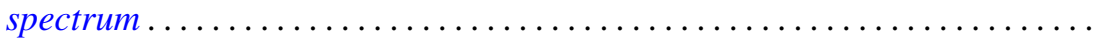

Raymond Frank Dickman and Alan Zame, Functionally compact spaces ...... 303

Ronald George Douglas and Walter Rudin, Approximation by inner

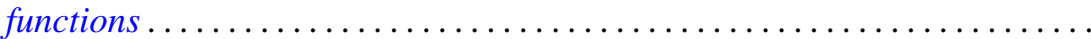

John Walter Duke, A note on the similarity of matrix and its conjugate

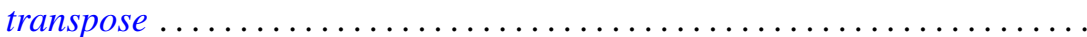

Micheal Neal Dyer and Allan John Sieradski, Coverings of mapping

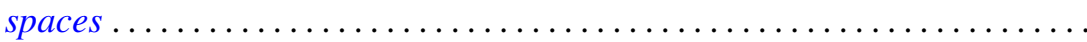

Donald Campbell Dykes, Weakly hypercentral subgroups of finite groups .....

Nancy Dykes, Mappings and realcompact spaces.....................

Edmund H. Feller and Richard Laham Gantos, Completely injective

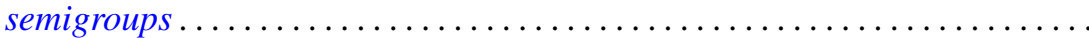

Irving Leonard Glicksberg, Semi-square-summable Fourier-Stieltjes

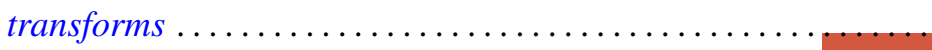

Samuel Irving Goldberg and Kentaro Yano, Integrability of almost cosymplectic structures...

Seymour Haber and Charles Freeman Osgood, On the sum $\sum\langle n \alpha\rangle^{-t}$ and numerical integration ..........................

Sav Roman Harasymiv, Dilations of rapidly decreasing functions ....

William Leonard Harkness and R. Shantaram, Convergence of a sequence of

transformations of distribution functions

Herbert Frederick Kreimer, Jr., A note on the outer Galois theory of rings ...

James Donald Kuelbs, Abstract Wiener spaces and applications to analysis. .

Roland Edwin Larson, Minimal $T_{0}$-spaces and minimal $T_{D}$-spaces...

A. Meir and Ambikeshwar Sharma, On Ilyeff's conjecture .

Isaac Namioka and Robert Ralph Phelps, Tensor products of compact convex sets....

James L. Rovnyak, On the theory of unbounded Toeplitz operators ....

Benjamin L. Schwartz, Infinite self-interchange graphs.......

George Szeto, On the Brauer splitting theorem...

Takayuki Tamura, Semigroups satisfying identity $x y=f(x$,

Kenneth Tolo, Factorizable semigroups .................. 